\title{
Electronic Coupling and Thermal Relaxation in Self-assembled InAs Quantum Dot Superlattices
}

\author{
E. Petitprez, N.T. Moshegov, E. Marega Jr., P. Basmaji, \\ Departamento de Física e Ciência dos Materiais - IFSC - USP \\ Caixa Postal 369 - 13560-970 São Carlos, SP, Brazil
}

A. Mazel, D. Dorignac, and R. Fourmeaux

CEMES/CNRS, BP 434731055 Toulouse Cedex 04, France

Received February, 1998

\begin{abstract}
We report optical and structural characterizations of InAs quantum dot superlattices grown by molecular beam epitaxy on GaAs (001). Cross-sectional electron microscopy imaging reveals $\sim 20$ $\mathrm{nm}$ diameter In As islands well aligned along the growth direction. Low temperature photoluminescence spectra exhibit a clear redshift with decreasing island vertical separation, as a result of electronic coupling between stacked dots, given that the larger shift is obtained for $70 \AA$ spacing. At high excitation power, the photoluminescence spectra show a doublet structure corresponding to coupled and uncoupled quantum dot states. The temperature dependence of the photoluminescence indicates that thermionic emission activation energy is much lower than the estimated barrier height. Such a difference is explained by the presence of non-radiative recombination centers due to strain relaxation and In segregation.
\end{abstract}

\section{Introduction}

Self-organized semiconductor growth has recently gained considerable interest due to the possibilities it offers as an alternative formation of quantum dot (QD) structures, avoiding post-growth processing techniques drawbacks. QD arrays produced in this way have been extensively investigated by optical[1-3], structural[4,5] and electrical[6,7] measurements, showing their potentiality to act as optoelectronic devices. Although QD laser operation has already been demonstrated[8-10], the degree of uniformity achieved in single layer QD structures still leads to a significant gain bandwidth broadening[11]. The growth of QD superlattices has then been considered as a way to increase QD arrays uniformity. Such structures surprisingly exhibit a vertical self-organization, since new islands tend to nucleate directly above the buried ones due to local surface strain minima[12,13]. Recent results also suggest that QD stacking leads to more uniform island size distribution[14,15]. Moreover, the formation of vertically ordered QD stacks allows to exploit electronic coupling of the islands by controlling the inter island separation thickness[16]. In this paper we report on photoluminescence (PL) investigations of InAs QD superlattices (SL) grown by molecular beam epitaxy (MBE) with differ- ent GaAs spacer layer thicknesses. Transmission electron microscopy (TEM) is used to image QD vertical alignment. The optical results suggest electronic coupling is best achieved for a given spacer layer thickness. For this specific thickness, coupled and uncoupled electronic states are observed. Finally, thermal relaxation is found to occur via non-radiative defects centers.

\section{Growth and experimental de- tails}

The structures were grown by conventional molecular beam epitaxy on (100) GaAs substrates under an $\mathrm{As}_{4}$ background pressure of $2 \times 10^{-6} \mathrm{~Pa}$. InAs and GaAs rates were set to 0.1 and 0.35 monolayer $(\mathrm{ML}) / \mathrm{s}$, respectively. The whole growth process was controlled in situ by reflection high energy electron diffraction (RHEED). After oxide desorption, a $0.3 \mu \mathrm{m}$ thick GaAs buffer layer was grown at $580{ }^{\circ} \mathrm{C}$, followed by ten periods of an $\mathrm{AlAs}(5 \mathrm{ML}) / \mathrm{GaAs}(10 \mathrm{ML})$ superlattice, and then by a $0.1 \mu \mathrm{m}$ GaAs layer. Next, the substrate temperature was lowered to $450{ }^{\circ} \mathrm{C}$ and $3 \mathrm{InAs}$ ML were deposited, followed by a GaAs spacer layer. The spacer layer thickness ranged from 40 to $130 \AA$. This sequence was repeated 3 and 7 times to obtain the multilayered 
structures. Variations of the RHEED specular beam intensity at In As growth initial stages indicated the formation of a complete InAs monolayer. At $1.8 \mathrm{ML}$ InAs coverage, spots appeared on the RHEED pattern due to $3 \mathrm{D}$ island formation, followed by a rapid fall-off of the specular beam intensity. During GaAs spacer layer deposition, the specular beam intensity first did not vary and then gradually increased, presenting superimposed oscillations. Meanwhile, the $3 \mathrm{D}$ island spots slowly disappeared, revealing the $(2 \times 4)$ GaAs surface reconstruction pattern. The structure was finally capped with a $500 \AA$ A GaAs layer. A single QD layer sample was also grown with the same conditions for reference purpose.

The samples were prepared for cross-sectional TEM observations by conventional mechanical polishing and ion milling techniques, and then observed with a PHILIPS CM30ST microscope under an acceleration voltage of $300 \mathrm{kV}$. PL investigations were performed in a closed cycle He cryostat under $\mathrm{Ar}^{+}$laser green line excitation $(514.5 \mathrm{~nm})$. The optical emission spectra were analyzed by a $0.5 \mathrm{~m}$ single grating spectrometer and measured in lock-in mode by a photomultiplier.

\section{Results and discussion}

Fig. 1 presents a cross-sectional TEM image of a 3 layer QD superlattice sample taken along the [2] zone axis. The spacer layer is $50 \AA$. The strain contrast clearly reveals $\sim 20 \mathrm{~nm}$ diameter lens-shaped InAs island formation for all layers, confirming RHEED observations. No structural defects, such as QD non-formation, microtwins or dislocations could be observed. As expected, QDs align vertically in stacks. Moreover, in some cases, QDs in the upper layer seem larger than those in the first layer. Since QD size modification has a considerable influence on PL peak position, it is therefore important to determine whether such phenomenon really occurs. Unfortunately, due to rather thick samples, high resolution TEM images did not permit to determine precisely QD lateral sizes.

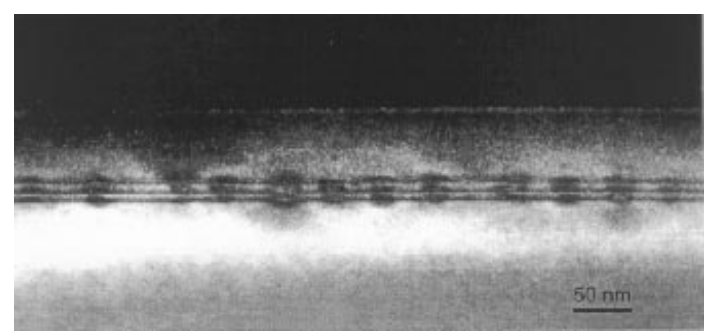

Figure 1. Dark-field $(g=200)$ cross-sectional TEM micrograph of a 3-layer QD SL sample.

Fig. 2 shows measured PL spectra for 3-layer QD superlattices with 130,100 and $70 \AA$ GaAs spacer lay- ers. A single QD layer PL spectrum is also presented for comparison. The main peak is due to electron-hole QD ground state recombination. Stacking a few QD layers slightly redshifts the PL peak. Decreasing spacer layer thickness down to $70 \AA$ also clearly drives the optical emission towards lower energies. Stacking induced strain relaxation accounts for part of this band gap reduction. Additionally, wave-function overlap allows electronic coupling between the islands, and thus lowers the QD ground state energy level. Closer islands are expected to produce stronger coupling, hence shifting the PL peak towards lower energies for samples with thinner spacer layers. Fig. 3 summarizes PL peak position behavior as a function of GaAs spacer layer thickness for the two series of samples. As discussed earlier, PL spectrum experiments a redshift as the spacer layer is reduced from 130 to $70 \AA$ due to electronic coupling between nearby dots. Surprisingly, as the spacer layer further decreases, the optical emission is blue-shifted. We therefore conclude there exists a certain spacer layer thickness for which coupling is best attained, and that this thickness is about $70 \AA$. We suggest that strain relaxation in too closely stacked islands allows QD lateral size modification along the vertical direction, what, in turn, inhibits coupled states formation.

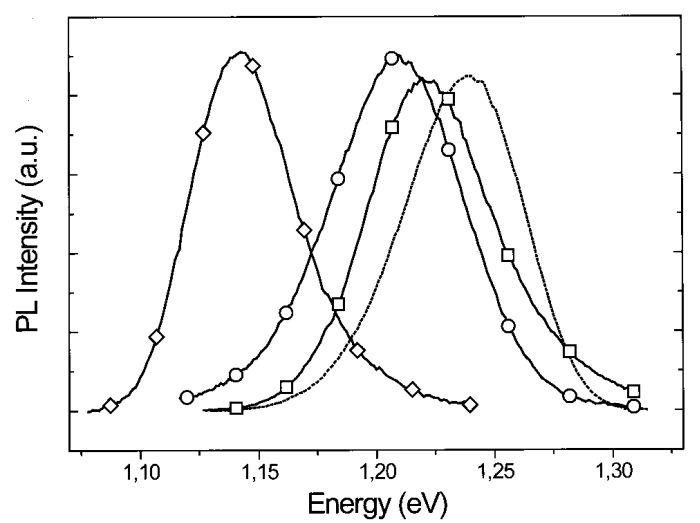

Figure 2. PL spectra of a single QD layer (dotted line) and of 3-layer QD SL samples with 130 (squares), 100 (circles) and $70 \AA$ (diamonds) GaAs spacer layers.

The optical properties of the samples were then investigated as a function of the excitation density. All samples exhibited gaussian-shaped PL spectra in the whole excitation power range, except the two samples with a $70 \AA$ thick spacer. Fig. 4 shows the excitation density dependence of one of these samples. At low excitation, PL spectrum is dominated by the 1.150 $\mathrm{eV}$ peak. At higher excitation densities, the spectrum exhibits a doublet structure that can be fitted by two gaussian peaks. Further increasing the excitation density leads the second peak, located around $1.20 \mathrm{eV}$, to 
dominate the spectrum. A linear dependence with excitation density is derived for both peaks, hence we do not attribute any of them to an impurity-assisted radiative recombination. Furthermore, these two peaks cannot arise from different QD size distribution since both of them should be observable with the same intensity ratio in the whole excitation density range.

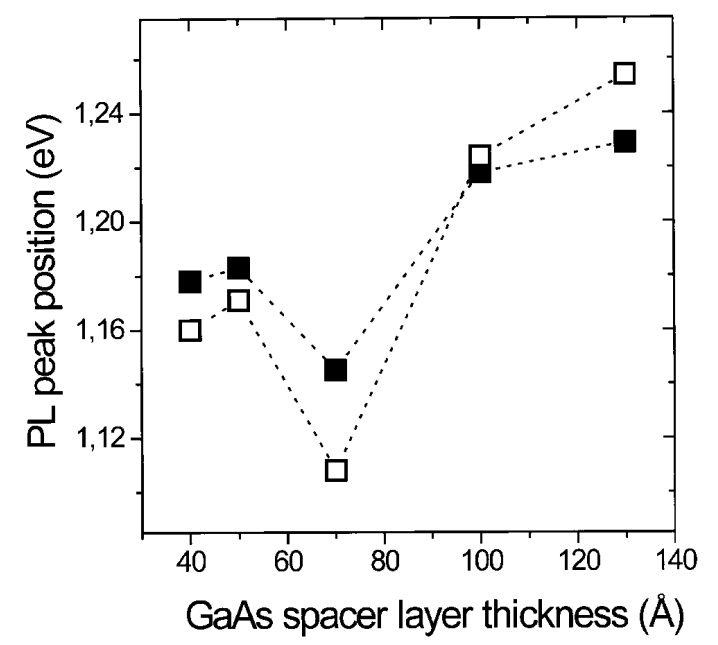

Figure 3. PL peak position as a function of GaAs spacer layer thickness. Solid and open squares refer to 3 - and 7layer QD superlattices, respectively.

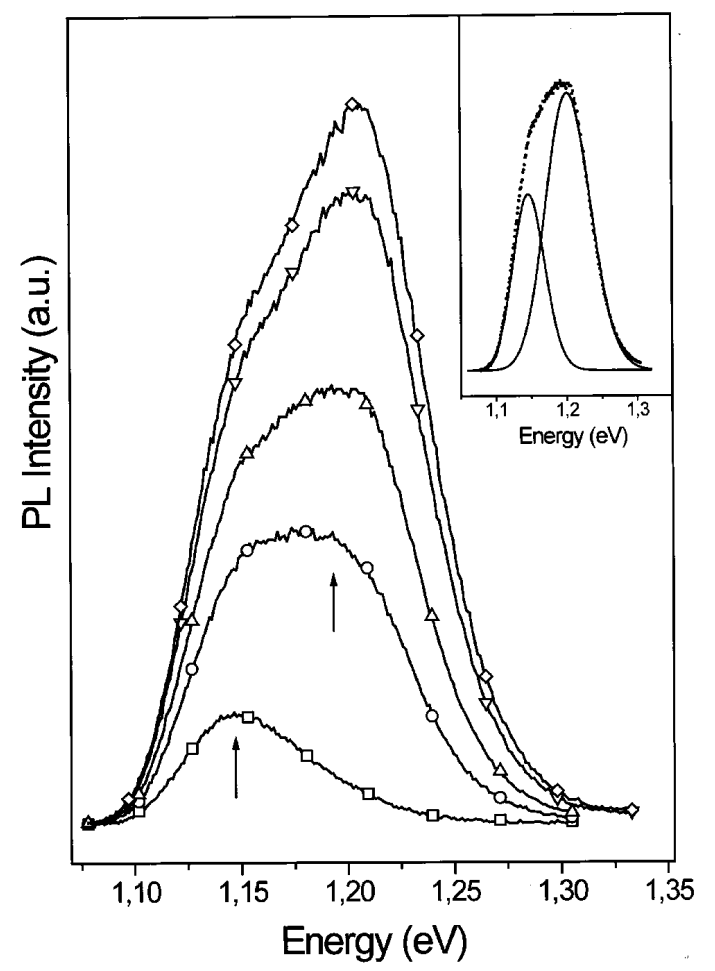

Figure 4. Excitation density influence on a 3-layer QD SL sample with $70 \AA$ spacer layer. Excitation density was 10 (squares), 30 (circles), 65 (up triangles), 130 (down triangles), and 190 (diamonds) $\mathrm{KW} / \mathrm{cm}^{2}$. Inset shows the two gaussian components used for fitting.
In multilayered samples, the apparition of new PL peaks with increasing excitation density has been attributed to optical emission from excited states[17]. For a single set of $20 \mathrm{~nm}$ diameter QDs, theoretical and experimental results show that the first excited state transition occurs 50 to $80 \mathrm{meV}$ above the ground state transition[18,19]. As the energy difference between the two peaks of fig. 4 is about $50 \mathrm{meV}$, optical recombination from excited states could be responsible for the PL peak doublet structure. However, in closely stacked QD layers, vertical electronic coupling between nearby dots has to be considered. The calculated ground state energy lowering due to such coupling ranges between 20 and $50 \mathrm{meV}[20,21]$, and the first excited state, even lowered by the coupling, lies well above the uncoupled ground state[20]. We therefore attribute these low- and high-energy peaks to coupled an uncoupled QDs, respectively. As in Ref. [22], we suppose uncoupled states arise from QD lateral size variations within the vertical stack. Coupled states, due to their lower ground state, are first populated. With increasing excitation density, all coupled states become populated and radiative recombination also occurs via uncoupled states. At high excitation densities, this recombination process dominates due to the lower density of coupled QD states.

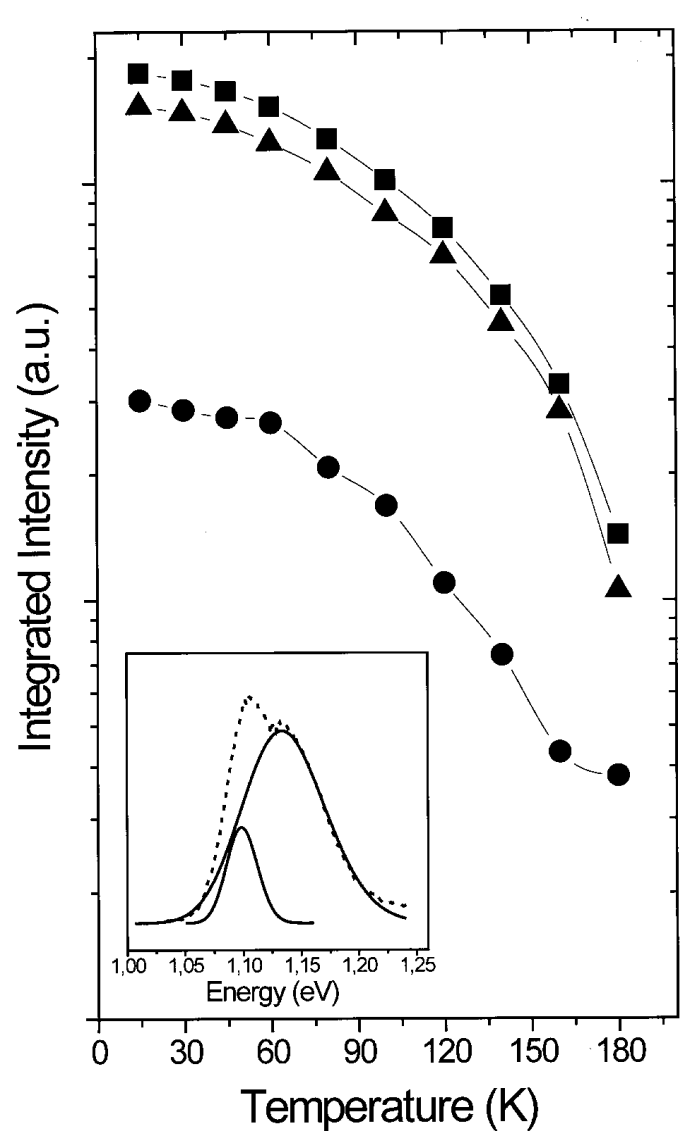

Figure 5. Wavelength-integrated PL intensity temperature dependence of the 7-layer QD SL sample with $70 \AA$ thick spacer. Squares, circles and triangles refer to, respectively, whole PL spectrum, low-energy gaussian component and 
high-energy gaussian component. The inset shows the two gaussian components at $15 \mathrm{~K}$.

PL measurements as a function of temperature were also carried out to investigate thermal relaxation processes in these samples. All samples exhibited a good thermal stability and a good PL signal could be detected up to $180 \mathrm{~K}$. Fig 5 presents integrated PL intensity temperature dependence of a 7-layer QD SL. The two gaussian components exhibit the same temperature stability, but slightly different behavior because of the different degree of wave function localization in the coupled and uncoupled states. From these plots were extracted the PL quenching activation energies. For low- and high energy peak, respectively, we obtained 38 and $78 \mathrm{meV}$, leading to a $40 \mathrm{meV}$ difference, which is very close to the $36 \mathrm{meV}$ PL peak position difference (see fig. 5 inset). PL quenching process is therefore the same for coupled and uncoupled states. However, these values are much smaller than the estimated InAsGaAs barrier height[23]. Similarly, the obtained values ranged from 30 to $108 \mathrm{meV}$ for all samples. We therefore suppose that PL quenching does not arise from carrier thermionic emission to the GaAs barrier but from non-radiative recombination centers. Such centers may originate from strain-induced structural defects, as well as from interface defects due to In segregation.

\section{Conclusion}

We have investigated electronic coupling and thermal relaxation in QD superlattices with different GaAs spacer layer thicknesses by PL measurements. The ground-state transition energy of the stacked QDs is found to decrease with decreasing spacer thickness as a result of the higher degree of strain relaxation and electronic coupling. For very closely stacked QDs, strain relaxation inhibits electronic coupling by modifying island lateral size. PL quenching activation energies are found to be considerably smaller than the estimated barrier height, indicating that thermal quenching occurs through carrier non-radiative recombination via defect states.

\section{Acknowledgments}

This work is supported by the Fundação de Amparo à Pesquisa do Estado de São Paulo (FAPESP) and the Conselho Nacional de Desenvolvimento Científico e Tecnológico (CNPq), Brazil.

\section{References}

[1] S. Fafard, R. Leon, D. Leonard, J.L. Merz, and P.M. Petroff, Superlatt. Microstruc. 16, 303 (1994).

[2] D. Leonard, M. Krishnamurthy, C.M. Reaves, S.P. Denbaars, and P.M. Petroff, Appl. Phys. Lett. 63, 3203 (1993).
[3] D. Leonard, S. Fafard, K. Pond, Y.H. Zhang, J.L. Merz, and P.M. Petroff, J. Vac. Sci. Technol. B 12, 2516 (1994).

[4] M. Grundmann, J. Christen, N.N. Ledentsov, J. Bohrer, D. Bimberg, S.S. Ruvimov, P. Werner, U. Richter , U. Gosele, J. Heydenreich, V.M. Ustinov, A.Y. Egorov, A.E. Zhukov, P.S. Kopev, and Z.I. Alferov, Phys. Rev. Lett. 74, 4043 (1995).

[5] R. Notzel, T. Fukui, H. Hasegawa, J. Temmyo, A. Kozen, and T. Tamamura, Chem. Vap. Dep. 1, 81 (1995).

[6] H. Drexler, D. Leonard, W. Hansen, J.P. Kotthaus, and P.M. Petroff, Phys. Rev. Lett. 73, 2252 (1994).

[7] G. Medeiros-Ribeiro, D. Leonard, and P.M. Petroff, Appl. Phys. Lett. 66, 1767 (1995).

[8] R. Mirin, A.C. Gossard, and J.E. Bowers, Physica E 2, 738 (1998).

[9] K. Kamath, N. Chervela, K.K. Linder, T. Sosnowski, H.T. Jiang, T. Norris, J. Singh, and P. Bhattacharya, Appl. Phys. Lett. 71, 927 (1997).

[10] N. Kirstaedter, O.G. Schmidt, N.N. Ledentsov, D. Bimberg, V.M. Ustinov, A.Y. Egorov, A.E. Zhukov, M.V. Maximov, P.S. Kopev, and Z.I. Alferov, Appl. Phys. Lett. 69, 1226 (1996).

[11] M. Grundmann and D. Bimberg, Jap. J. Appl. Phys. Part 1 36, 4181 (1997).

[12] Q. H. Xie, P. Chen, and A. Madhukar, Appl. Phys. Lett. 65, 2051 (1994).

[13] Q. H. Xie, A. Madhukar, P. Chen, and N.P. Kobayashi, Phys. Rev. Lett. 75, 2542 (1995).

[14] J. Tersoff, C. Teichert, and M.G. Lagally, Phys. Rev. Lett. 76, 1675 (1996).

[15] G. S. Solomon, S. Komarov, J.S. Harris, and Y. Yamamoto, J. Cryst. Growth 175, 707 (1997).

[16] G. S. Solomon, J.A. Trezza, A.F. Marshall, and J.S. Harris, Phys. Rev. Lett. 76, 952 (1996).

[17] Q. H. Xie, N.P. Kobayashi, T.R. Ramachandran, A. Kalburge, P. Chen, and A. Madhukar, J. Vac. Sci. Technol. B14, 2203 (1996).

[18] M. Grundmann, N.N. Ledentsov, O. Stier, J. Bohrer, D. Bimberg, V.M. Ustinov, P.S. Kopev, and Z.I. Alferov, Phys. Rev. B 53, 10509 (1996).

[19] K. H. Schmidt, G. Medeiros-Ribeiro, M. Oestreich, P.M. Petroff, and G.H. Dohler, Phys. Rev. B 54, 11346 (1996).

[20] L. C. Fonseca, J.L. Jimenez, and J.P. Leburton, Phys. Rev. B58, 9955 (1998).

[21] M. Colocci, A. Vinattieri, L. Lippi, F. Bogani, M. RosaClot, S. Taddei, A. Bosacchi, S. Franchi, and P. Frigeri, Appl. Phys. Lett. 74, 564 (1999).

[22] R. Heitz, A. Kalburge, Q. Xie, M. Grundmann, P. Chen, A. Hoffmann, A. Madhukar, and D. Bimberg, Phys. Rev. B 57, 9050 (1998).

[23] M. Grundmann, O. Stier, and D. Bimberg, Phys. Rev. B 52, 11969 (1995). 